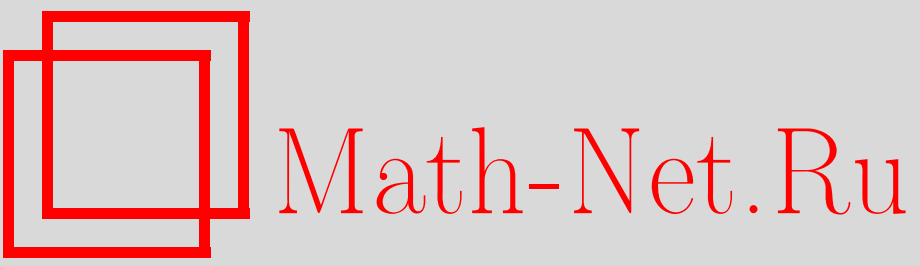

Л. Н. Куртова, Н. Н. Мотькина, О видах решений задачи Лагранжа, Итоги науки и техн. Сер. Соврем. мат. и ее прил. Темат. обз., 2019, том 166, 41-48

DOI: https://doi.org/10.36535/0233-6723-2019-166-41-48

Использование Общероссийского математического портала Math-Net.Ru подразумевает, что вы прочитали и согласны с пользовательским соглашением

http://www.mathnet.ru/rus/agreement

Параметры загрузки:

IP: 18.234 .156 .22

26 апреля 2023 г., 08:32:06 


\title{
О ВИДАХ РЕШЕНИЙ ЗАДАЧИ ЛАГРАНЖА
}

\author{
(c) 2019 г. $\quad$ Л. Н. КУРТОВА, Н. Н. МОТЬКИНА
}

\begin{abstract}
АннотАция. Представлен разбор некоторых случаев, когда натуральное число не представимо диагональной квадратичной формой с четырьмя целыми переменными.
\end{abstract}

Ключевъе слова: квадратичная форма, тригонометрическая сумма, сумма Гаусса, сравнение, сумма Клоостермана, асимптотическая формула.

\section{ON TYPES OF SOLUTIONS OF THE LAGRANGE PROBLEM}

\author{
(C) 2019 L. N. KURTOVA, N. N. MOT'KINA
}

\begin{abstract}
In this paper, we present an analysis of some cases where a positive integer cannot be represented by a diagonal quadratic form with four integer variables.

Keywords and phrases: quadratic form, trigonometric sum, Gauss sum, comparison, Kloosterman sum, asymptotic formula.
\end{abstract}

AMS Subject Classification: 11D09

1. Введение. В литературе по теории чисел известно много задач о представлении натурального числа в виде сумм различного вида. Одна из них задача Лагранжа (1770 г.) о том, что любое целое положительное число является суммой не более четырех квадратов натуральных чисел

$$
l_{1}^{2}+l_{2}^{2}+l_{3}^{2}+l_{4}^{2}=N .
$$

До этой задачи математики П. Ферма, Л. Эйлер и другие изучали квадратичные формы частного вида. Ж. Лагранж установил точную связь между вопросом о представлении чисел квадратичной формой и разрешимостью соответствующего сравнения второй степени.

К. Ф. Гаусс, а затем Л. Дирихле, продолжая исследования Эйлера, создали теорию представления натуральных чисел квадратичными формами. Гаусс ввел так называемые суммы Гаусса

$$
S(q, a, b)=\sum_{1 \leqslant l \leqslant q} e^{2 \pi i\left(a l^{2}+b l\right) / q},
$$

которые явились первыми примерами тригонометрических сумм, и показал их полезность в решении задач теории чисел.

В 1926 г. Х. Клоостерман обобщил задачу Лагранжа (см. [5]), рассмотрев вопрос о представлении целого положительного числа в виде диагональной квадратичной формы, зависящей от четырех целых переменных (задача Клоостермана):

$$
n=a x^{2}+b y^{2}+c z^{2}+d t^{2} .
$$

Он получил для числа решений $r(n)$ уравнения (1) асимптотическую формулу

$$
r(n)=\frac{\pi^{2}}{\sqrt{a b c d}} n S(n)+O\left(n^{17 / 18+\varepsilon}\right)
$$


где

$$
S(n)=\sum_{q=1}^{\infty} \frac{1}{q^{4}} \sum_{\substack{l=1 \\(l, q)=1}}^{q} e^{-2 \pi i n l / q} S(q, a l, 0) S(q, b l, 0) S(q, c l, 0) S(q, d l, 0) .
$$

Кроме того, в [5] были рассмотрены некоторые примеры, когда число представлений равно нулю. Их доказательство было основано на теории сравнений, а в отдельных случаях отсутствовало. Вопрос о представлении четного числа рассмотрен Клоостерманом более подробно, чем случаи с нечетным простым $p$, входящим в разложение $n$.

Применение точных формул для сумм Гаусса и Рамануджана (см. [1-4]) позволило подробно рассмотреть случаи для нечетного простого $p$, при которых уравнение (1), где $a, b, c, d, n-$ положительные целые числа, не имеет решения, и дополнить их.

2. Основные результаты. Пусть $p$ - нечетное простое число, $a, b, c, d, n-$ положительные целые.

Теорема 1. Уравнение $n=a x^{2}+b y^{2}+c z^{2}+d t^{2}$ не имеет решения в следующих случаях:

1.1. если п и р взаимно просты, коэфбициенты $a, b, c, d$ делятся на р;

1.2. если п и р взаимно прость, три коэфбициента квадратичной бормз ах ${ }^{2}+b y^{2}+c z^{2}+d t^{2}$ делятся на р, произведение четвертого коэфбиииента и п является квадратичным невычетом по модулю $р$.

Теорема 2. Пусть

$$
\begin{gathered}
a=p^{\alpha_{1}} a_{1}, \quad\left(a_{1}, p\right)=1, \quad b=p^{\beta_{1}} b_{1}, \quad\left(b_{1}, p\right)=1, \\
(c, p)=1, \quad(d, p)=1, \quad n=p^{\eta_{1}} n_{1}, \quad\left(n_{1}, p\right)=1 .
\end{gathered}
$$

Уравнение $n=a x^{2}+b y^{2}+c z^{2}+d t^{2}$ не имеет решения в следуюших случаях:

2.1. если $\eta_{1}<\alpha_{1} \leqslant \beta_{1}, \eta_{1}$ - нечетное число, $(-c d)$ - квадратичный невычет по модулю $р$;

2.2. если $\eta_{1}=\alpha_{1}<\beta_{1}, \eta_{1}$ - нечетное число, $a_{1} n_{1} u(-c d)$ - квадратичные невычеты по модулю $p$

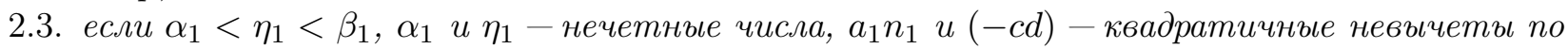
модулю р.

Теорема 3. Пусть

$$
\begin{gathered}
a=p^{\alpha_{1}} a_{1}, \quad\left(a_{1}, p\right)=1, \quad b=p^{\beta_{1}} b_{1}, \quad\left(b_{1}, p\right)=1, \\
c=p^{\gamma_{1}} c_{1}, \quad\left(c_{1}, p\right)=1, \quad(d, p)=1, \quad n=p^{\eta_{1}} n_{1}, \quad\left(n_{1}, p\right)=1 .
\end{gathered}
$$

Уравнение $n=a x^{2}+b y^{2}+c z^{2}+d t^{2}$ не имеет решения в следуюших случаях:

3.1. если $\eta_{1}<\alpha_{1} \leqslant \beta_{1} \leqslant \gamma_{1}$ и $\eta_{1}$ - нечетное число;

3.2. если $\eta_{1}<\alpha_{1} \leqslant \beta_{1} \leqslant \gamma_{1}, \eta_{1}$-четное число и $d n_{1}-$ чвадратичный невычет по модулю $p$;

3.3. если $\eta_{1}=\alpha_{1}<\beta_{1} \leqslant \gamma_{1}, \eta_{1}$ - нечетное число и $a_{1} n_{1}-$ квадратичный невычет по модулю $p$;

3.4. если $\alpha_{1}<\eta_{1}<\beta_{1} \leqslant \gamma_{1}, \eta_{1}$ - нечетное число, $\alpha_{1}$ - четное число и $\left(-a_{1} d\right)-$ квадратичный невычет по модулю р;

3.5. если $\alpha_{1}<\eta_{1}<\beta_{1} \leqslant \gamma_{1}, \alpha_{1}$-нечетное число $и$

$$
\left(\frac{d}{p^{\eta_{1}+1}}\right)\left(\frac{a_{1}}{p^{\eta_{1}}}\right)\left(\frac{n_{1}}{p}\right)=-1 ;
$$

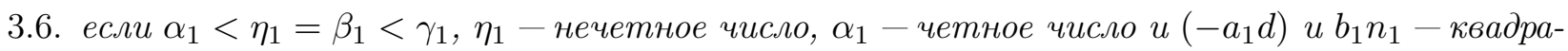
тичные невычеты по модулю $р$;

3.7. если $\alpha_{1} \leqslant \beta_{1}<\eta_{1}<\gamma_{1}$, $\eta_{1}$ - четное число, $\alpha_{1}$-нечетное число, $\beta_{1}$ - нечетное число, $\left(-a_{1} b_{1}\right)$ и dn $n_{1}-$ квадратичные невычеть по модулю $p$

3.8. если $\alpha_{1} \leqslant \beta_{1}<\eta_{1}<\gamma_{1}$, $\eta_{1}$ - нечетное число, $\alpha_{1}$-нечетное число, $\beta_{1}$ - четное число, $\left(-b_{1} d\right)$ и $a_{1} n_{1}-$ квадратичные невычеты по модулю $p$

3.9. если $\alpha_{1} \leqslant \beta_{1}<\eta_{1}<\gamma_{1}$, $\eta_{1}$ - нечетное число, $\alpha_{1}$-четное число, $\beta_{1}$ - нечетное число, $\left(-a_{1} d\right)$ и $b_{1} n_{1}-$ квадратичные невычеты по модулю $р$. 
Отметим, что утверждение теоремы 1 доказано в работе Клоостермана [5] с помощью теории сравнений. Случаи 2.1 и 2.2 приведены у Клоостермана без доказательства, а утверждение 2.3 является новым и не рассматривалось ранее. Результаты теоремы 3 являются новыми, не исследовались в работе Клоостермана.

Приведем подробное доказательство теоремы 1. Теоремы 2 и 3 доказываются аналогично. Нам понадобятся следующие утверждения, доказательство которых можно найти в [1].

\section{3. Вспомогательные леммы.}

Лемма 1 (равенства для суммы Гаусса).

1. $\operatorname{Eсли~}\left(q_{1}, q_{2}\right)=1$, mo

$$
S\left(q_{1} q_{2}, u, 0\right)=S\left(q_{1}, u q_{2}, 0\right) S\left(q_{2}, u q_{1}, 0\right) .
$$

2. $\operatorname{Eсли~}(q, 2 u)=1, m o$

$$
S(q, u, 0)=\left(\frac{u}{q}\right) S(q, 1,0)
$$

где $\left(\frac{u}{q}\right)-$ символ Якоби,

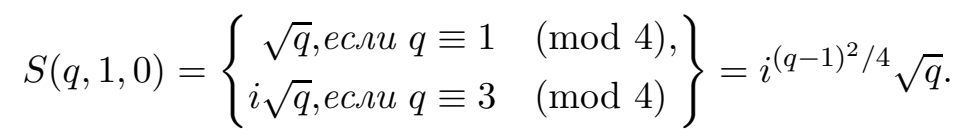

3. $\operatorname{Eсли~}(q, u)=n$, mo

$$
S(q, u, 0)=n S\left(\frac{q}{n}, \frac{u}{n}, 0\right) .
$$

Лемма 2 (равенства для суммы Рамануджана). Пусть

$$
K(q, u)=\sum_{\substack{1 \leqslant l \leqslant q \\(l, q)=1}} e^{2 \pi i u l / q}
$$

- сумма Рамануджсана. Справедливы следующие утверждения.

1. $K(q,-u)=K(q, u)$.

2. При $\left(q_{1}, q_{2}\right)=1$ имеет место равенство

$$
K\left(q_{1} q_{2}, u\right)=K\left(q_{1}, u_{1}\right) K\left(q_{2}, u_{2}\right),
$$

где $u_{1}$ и $u_{2}$ определены по модулям $q_{1}$ и $q_{2}$ соответственно сравнением

$$
u_{1} q_{2}+u_{2} q_{1} \equiv u \quad\left(\bmod q_{1} q_{2}\right) .
$$

3. Пусть $(u, p)=1, \alpha>1$. Тогда

$$
K(p, u)=-1, \quad K\left(p^{\alpha}, u\right)=0 .
$$

4. Пусть $u=p^{\alpha} u_{1},\left(u_{1}, p\right)=1, \alpha>1, s>1$. Тогда

$$
K\left(p^{\alpha}, u\right)=p^{\alpha-1}(p-1), \quad K\left(p^{\alpha+1}, u\right)=-p^{\alpha}, \quad K\left(p^{\alpha+s}, u\right)=0 .
$$

Лемма 3 (равенства для обобщенной суммы Рамануджана). Пусть $p$ - нечетное простое число и

$$
K_{p}\left(p^{\alpha}, u\right)=\sum_{\substack{l=1 \\\left(l, p^{\alpha}\right)=1}}^{p^{\alpha}}\left(\frac{l}{p}\right) e^{2 \pi i u l / p^{\alpha}}
$$

- обобщенная сумма Рамануджана. Справедливы следующие утверждения.

1. Пусть $(u, p)=1, \alpha>1$. Тогда

$$
K_{p}(p, u)=S(p, u, 0), \quad K_{p}\left(p^{\alpha}, u\right)=0 .
$$

2. Пусть $u=p^{\alpha} u_{1},\left(u_{1}, p\right)=1, \alpha>1, s>1$. Тогда

$$
K_{p}\left(p^{\alpha}, u\right)=0, \quad K_{p}\left(p^{\alpha+1}, u\right)=p^{\alpha} S\left(p, u_{1}, 0\right), \quad K_{p}\left(p^{\alpha+s}, u\right)=0 .
$$


4. Доказательство теоремы 1. Для особого ряда $S(n)$ асимптотической формулы (2) рассмотрим функцию

$$
\Phi(q)=\frac{1}{q^{4}} \sum_{\substack{l=1 \\(l, q)=1}}^{q} e^{-2 \pi i n l / q} S(q, a l, 0) S(q, b l, 0) S(q, c l, 0) S(q, d l, 0) .
$$

Покажем, что она является мультипликативной. Пусть $q=q_{1} q_{2},\left(q_{1}, q_{2}\right)=1, l=l_{1} q_{2}+l_{2} q_{1}$; тогда из утверждения 1.1 леммы 1 следует, что

$$
S(q, a l, 0)=S\left(q_{1} q_{2}, a l_{1} q_{2}+a l_{2} q_{1}, 0\right)=S\left(q_{1}, a l_{1} q_{2}^{2}, 0\right) S\left(q_{2}, a l_{2} q_{1}^{2}, 0\right) .
$$

Учтем равенство

$$
S\left(q_{1}, a l_{1} q_{2}^{2}, 0\right)=\sum_{1 \leqslant j \leqslant q_{1}} e^{2 \pi i a l_{1} q_{2}^{2} j^{2} / q_{1}}=\sum_{q_{2} \leqslant j_{1} \leqslant q_{1} q_{2}} e^{2 \pi i a l_{1} j_{1}^{2} / q_{1}}=S\left(q_{1}, a l_{1}, 0\right) .
$$

Тогда

$$
S(q, a l, 0)=S\left(q_{1}, a l_{1}, 0\right) S\left(q_{2}, a l_{2}, 0\right) .
$$

Аналогичные рассуждения справедливы и для остальных сумм $S(q, b l, 0), S(q, c l, 0), S(q, d l, 0)$. Кроме того, из утверждения 2.2 леммы 2 следует, что

$$
\sum_{\substack{l=1 \\\left(l, q_{1} q_{2}\right)=1}}^{q_{1} q_{2}} e^{-2 \pi i n l /\left(q_{1} q_{2}\right)}=\sum_{\substack{l_{1}=1 \\\left(l_{1}, q_{1}\right)=1}}^{q_{1}} e^{-2 \pi i n l_{1} / q_{1}} \sum_{\substack{l_{2}=1 \\\left(l_{2}, q_{2}\right)=1}}^{q_{2}} e^{-2 \pi i n l_{2} / q_{2}} .
$$

Следовательно,

$$
\begin{gathered}
\Phi\left(q_{1} q_{2}\right)=\frac{1}{q_{1}^{4}} \sum_{\substack{l_{1}=1 \\
\left(l_{1}, q_{1}\right)=1}}^{q_{1}} e^{-2 \pi i n l_{1} / q_{1}} S\left(q_{1}, a l_{1}, 0\right) S\left(q_{1}, b l_{1}, 0\right) S\left(q_{1}, c l_{1}, 0\right) S\left(q_{1}, d l_{1}, 0\right) \times \\
\times \frac{1}{q_{2}^{4}} \sum_{\substack{l_{2}=1 \\
\left(l_{2}, q_{2}\right)=1}}^{q_{2}} e^{-2 \pi i n l_{2} / q_{2}} S\left(q_{2}, a l_{2}, 0\right) S\left(q_{2}, b l_{2}, 0\right) S\left(q_{2}, c l_{2}, 0\right) S\left(q_{2}, d l_{2}, 0\right) .
\end{gathered}
$$

Таким образом, $\Phi\left(q_{1} q_{2}\right)=\Phi\left(q_{1}\right) \Phi\left(q_{2}\right)$, и мультипликативность доказана.

По свойству мультипликативной функции получим представление особого ряда в виде произведения

$$
\sum_{q=1}^{+\infty} \Phi(q)=\prod_{p \mid q}\left(1+\Phi(p)+\Phi\left(p^{2}\right)+\ldots\right) .
$$

Получим точные формулы для всевозможных произведений при нечетном простом $p,(n, p)=1$.

4.1. Случай, когда $a, b, c, d, n$ взаимно просты с $p$. Пусть

$$
(a, p)=1, \quad(b, p)=1, \quad(c, p)=1, \quad(d, p)=1, \quad(n, p)=1 .
$$

Тогда для произведения сумм Гаусса (лемма 1, утверждение 1.2) получаем формулу

$$
S\left(p^{\alpha}, a l, 0\right) S\left(p^{\alpha}, b l, 0\right) S\left(p^{\alpha}, c l, 0\right) S\left(p^{\alpha}, d l, 0\right)=\left(\frac{a b c d}{p^{\alpha}}\right) p^{2 \alpha} .
$$

Следовательно,

$$
\Phi\left(p^{\alpha}\right)=\left(\frac{a b c d}{p^{\alpha}}\right) \frac{1}{p^{2 \alpha}} K\left(p^{\alpha},-n\right) .
$$

Используя равенство 3 из леммы 3.2 , получаем

$$
\Phi(p)=-\left(\frac{a b c d}{p}\right) \frac{1}{p^{2}}, \quad \Phi\left(p^{\alpha}\right)=0, \quad \alpha>1 .
$$


Получаем первый множитель в представлении особого ряда в виде произведений по простым числам:

$$
\prod_{\substack{p:(a, p)=1,(b, p)=1,(c, p)=1,(d, p)=1,(n, p)=1}}\left(1-\left(\frac{a b c d}{p}\right) \frac{1}{p^{2}}\right) .
$$

Отметим, что при $(a, p)=1,(b, p)=1,(c, p)=1,(d, p)=1, n=p^{\alpha} n_{1},\left(n_{1}, p\right)=1, \alpha>1$ формулы для произведения сумм Гаусса аналогичны:

$$
\Phi\left(p^{\alpha}\right)=\left(\frac{a b c d}{p^{\alpha}}\right) \frac{1}{p^{2 \alpha}} K\left(p^{\alpha},-n\right) .
$$

Изменятся формулы для суммы Рамануджана. Используя равенство 4 из леммы 2, получаем, что

$$
\begin{gathered}
\Phi\left(p^{k}\right)=\left(\frac{a b c d}{p^{k}}\right) \frac{p-1}{p^{k+1}}, \quad k=1,2, \ldots, \alpha, \\
\Phi\left(p^{\alpha+1}\right)=-\left(\frac{a b c d}{p^{\alpha+1}}\right) \frac{1}{p^{\alpha+2}}, \quad \Phi\left(p^{\alpha+s}\right)=0, \quad s>1 .
\end{gathered}
$$

Получаем второй множитель в представлении особого ряда в виде произведений по простым числам:

$$
\prod_{\substack{(a, p)=1,(b, p)=1,(c, p)=1,(d, p)=1 \\ n=p^{\alpha} n_{1}, \alpha>1,\left(n_{1}, p\right)=1}}\left(1+\left(\frac{a b c d}{p}\right) \frac{p-1}{p^{2}}+\left(\frac{a b c d}{p^{2}}\right) \frac{p-1}{p^{3}}+\cdots+\left(\frac{a b c d}{p^{\alpha}}\right) \frac{p-1}{p^{\alpha+1}}-\left(\frac{a b c d}{p^{\alpha+1}}\right) \frac{1}{p^{\alpha+2}}\right) .
$$

В результате при $(a ; p)=(b ; p)=(c ; p)=(d ; p)=1, n=p^{\alpha} n_{1},\left(n_{1}, p\right)=1, \alpha \geqslant 1$ имеем

$$
\begin{aligned}
& \prod_{p}\left(1-\left(\frac{a b c d}{p}\right) \frac{1}{p^{2}}\right)\left(1+\left(\frac{a b c d}{p}\right) \frac{1}{p}+\left(\frac{a b c d}{p^{2}}\right) \frac{1}{p^{2}}+\cdots+\left(\frac{a b c d}{p^{\alpha}}\right) \frac{1}{p^{\alpha}}\right) \cdot . \\
& (a, p)=1,(b, p)=1 \\
& (c, p)=1,(d, p)=1 \\
& n=p^{\alpha} n_{1}, \alpha \geqslant 1 \\
& \left(n_{1}, p\right)=1
\end{aligned}
$$

Если $a b c d-$ квадратичный вычет по модулю $p$, то

$$
\begin{gathered}
1-\left(\frac{a b c d}{p}\right) \frac{1}{p^{2}}=1-\frac{1}{p^{2}}>3 / 4 \\
\left(1+\left(\frac{a b c d}{p}\right) \frac{1}{p}+\left(\frac{a b c d}{p^{2}}\right) \frac{1}{p^{2}}+\cdots+\left(\frac{a b c d}{p^{\alpha}}\right) \frac{1}{p^{\alpha}}\right)=\frac{p^{\alpha+1}-1}{p^{\alpha}(p-1)}>1 .
\end{gathered}
$$

Если $a b c d-$ квадратичный невычет по модулю $p$, то

$$
\begin{gathered}
1-\left(\frac{a b c d}{p}\right) \frac{1}{p^{2}}=1+\frac{1}{p^{2}}>1 \\
\left(1+\left(\frac{a b c d}{p}\right) \frac{1}{p}+\left(\frac{a b c d}{p^{2}}\right) \frac{1}{p^{2}}+\cdots+\left(\frac{a b c d}{p^{\alpha}}\right) \frac{1}{p^{\alpha}}\right)=\frac{p^{\alpha+1}-(-1)^{\alpha+1}}{p^{\alpha}(p+1)}>\frac{1}{2} .
\end{gathered}
$$

4.2. Случай, когда один из коэффициентов $a, b, c, d$ делится на $p,(n, p)=1$. Пусть

$$
a=p^{\alpha_{1}} a_{1}, \quad\left(a_{1}, p\right)=1, \quad(b, p)=1, \quad(c, p)=1, \quad(d, p)=1, \quad(n, p)=1 .
$$

Найдем $\Phi(p)$ :

$$
\Phi(p)=\frac{1}{p^{3}}\left(\frac{b c d}{p}\right) S^{3}(p, 1,0) \sum_{\substack{l=1 \\(l, p)=1}}^{p}\left(\frac{l}{p}\right) e^{-2 \pi i n l / p} .
$$


Используя лемму 3 (утверждение 3.1 ), получаем

$$
\Phi(p)=\frac{1}{p^{3}}\left(\frac{-b c d n}{p}\right) S^{4}(p, 1,0)=\frac{1}{p}\left(\frac{-b c d n}{p}\right) .
$$

Пусть $1<\alpha \leqslant \alpha_{1}$. Тогда

$$
\Phi\left(p^{\alpha}\right)=\frac{1}{p^{3 \alpha}}\left(\frac{b c d}{p^{\alpha}}\right) S^{3}\left(p^{\alpha}, 1,0\right) \sum_{\substack{l=1 \\\left(l, p^{\alpha}\right)=1}}^{p^{\alpha}}\left(\frac{l}{p^{\alpha}}\right) e^{-2 \pi i n l / p^{\alpha}}
$$

Если $\alpha$-четное, то

$$
\sum_{\substack{l=1 \\\left(l, p^{\alpha}\right)=1}}^{p^{\alpha}}\left(\frac{l}{p^{\alpha}}\right) e^{-2 \pi i n l / p^{\alpha}}=K\left(p^{\alpha},-n\right)=0
$$

в силу леммы 2 о сумме Рамануджана (утверждение 2.3). Если $\alpha$-нечетное, то

$$
\sum_{\substack{l=1 \\\left(l, p^{\alpha}\right)=1}}^{p^{\alpha}}\left(\frac{l}{p^{\alpha}}\right) e^{-2 \pi i n l / p^{\alpha}}=K_{p}\left(p^{\alpha},-n\right)=0
$$

в силу леммы 3 об обобщенной сумме Рамануджана (утверждение 3.1 ). Тогда $\Phi\left(p^{\alpha}\right)=0$.

Пусть $\alpha>\alpha_{1} \geqslant 1$. В этом случае

$$
\Phi\left(p^{\alpha}\right)=p^{\alpha_{1}-4 \alpha}\left(\frac{b c d}{p^{\alpha}}\right)\left(\frac{a_{1}}{p^{\alpha-\alpha_{1}}}\right) S^{3}\left(p^{\alpha}, 1,0\right) S\left(p^{\alpha-\alpha_{1}}, 1,0\right) \sum_{\substack{l=1 \\\left(l, p^{\alpha}\right)=1}}^{p^{\alpha}}\left(\frac{l}{p^{2 \alpha-\alpha_{1}}}\right) e^{-2 \pi i n l / p^{\alpha}} .
$$

Имеем

$$
\sum_{\substack{l=1 \\\left(l, p^{\alpha}\right)=1}}^{p^{\alpha}}\left(\frac{l}{p^{2 \alpha-\alpha_{1}}}\right) e^{-2 \pi i n l / p^{\alpha}}=0
$$

для четного $\alpha_{1}$ в силу леммы 2 (утверждение 2.3 ), а для нечетного $\alpha_{1}$ в силу леммы 3 (утверждение 3ю1). Тогда $\Phi\left(p^{\alpha}\right)=0$.

Получаем следующий множитель:

$$
\prod_{\substack{p \\ a=p^{\alpha_{1}} a_{1},\left(a_{1}, p\right)=1,(b, p)=1,(c, p)=1,(d, p)=1,(n, p)=1}}\left(1+\left(\frac{-b c d n}{p}\right) \frac{1}{p}\right) .
$$

Данная скобка больше 1 , если $(-b c d n)$ - квадратичный вычет по модулю $p$; больше $1 / 2$ и стремится к 1 с ростом $p$, если $(-b c d n / p)=-1$.

4.3. Случай, когда два коэфбициента делятся на $p,(n, p)=1$. Пусть

$$
a=p^{\alpha_{1}} a_{1}, \quad\left(a_{1}, p\right)=1, \quad b=p^{\beta_{1}} b_{1}, \quad\left(b_{1}, p\right)=1, \quad(c, p)=1, \quad(d, p)=1, \quad(n, p)=1 .
$$

Имеем

$$
\Phi(p)=\frac{1}{p^{2}}\left(\frac{c d}{p}\right) S^{2}(p, 1,0) \sum_{\substack{l=1 \\(l, p)=1}}^{p} e^{-2 \pi i n l / p}=-\left(\frac{-c d}{p}\right) \frac{1}{p} .
$$

Пусть $\min \left(\alpha_{1}, \beta_{1}\right)=\alpha_{1}$.

4.3.1. При $1<\alpha \leqslant \alpha_{1}$ с учетом утверждения 2.3 леммы 2 имеем

$$
\Phi\left(p^{\alpha}\right)=\frac{1}{p^{2 \alpha}}\left(\frac{c d}{p^{\alpha}}\right) S^{2}\left(p^{\alpha}, 1,0\right) \sum_{\substack{l=1 \\\left(l, p^{\alpha}\right)=1}}^{p^{\alpha}} e^{-2 \pi i n l / p^{\alpha}}=0 .
$$


4.3.2. При $\alpha_{1}<\alpha \leqslant \beta_{1}$

$$
\Phi\left(p^{\alpha}\right)=p^{\alpha_{1}-4 \alpha}\left(\frac{c d}{p^{\alpha}}\right)\left(\frac{a_{1}}{p^{\alpha-\alpha_{1}}}\right) S^{2}\left(p^{\alpha}, 1,0\right) S\left(p^{\alpha-\alpha_{1}}, 1,0\right) \sum_{\substack{l=1 \\\left(l, p^{\alpha}\right)=1}}^{p^{\alpha}}\left(\frac{l}{p^{\alpha-\alpha_{1}}}\right) e^{-2 \pi i n l / p^{\alpha}} .
$$

Сумма Рамануджана

$$
\sum_{\substack{l=1 \\\left(l, p^{\alpha}\right)=1}}^{p^{\alpha}}\left(\frac{l}{p^{\alpha-\alpha_{1}}}\right) e^{-2 \pi i n l / p^{\alpha}}
$$

равна нулю. При четном $\alpha-\alpha_{1}$ это следует из утверждения 2.3 леммы 2 , при нечетном из утверждения 3.1 леммы 3. Поэтому $\Phi\left(p^{\alpha}\right)=0$.

4.3.3. При $\alpha_{1} \leqslant \beta_{1}<\alpha$

$$
\begin{aligned}
\Phi\left(p^{\alpha}\right)=p^{\alpha_{1}+\beta_{1}-4 \alpha}\left(\frac{c d}{p^{\alpha}}\right) & \left(\frac{a_{1}}{p^{\alpha-\alpha_{1}}}\right)\left(\frac{b_{1}}{p^{\alpha-\beta_{1}}}\right) \times \\
\times & S^{2}\left(p^{\alpha}, 1,0\right) S\left(p^{\alpha-\alpha_{1}}, 1,0\right) S\left(p^{\alpha-\beta_{1}}, 1,0\right) \sum_{\substack{l=1 \\
\left(l, p^{\alpha}\right)=1}}^{p^{\alpha}}\left(\frac{l}{p^{2 \alpha-\alpha_{1}-\beta_{1}}}\right) e^{-2 \pi i n l / p^{\alpha}} .
\end{aligned}
$$

Как и в случае 4.3 .2 имеем $\Phi\left(p^{\alpha}\right)=0$. Получаем множитель

$$
\begin{aligned}
& \prod_{p}\left(1-\left(\frac{-c d}{p}\right) \frac{1}{p}\right) . \\
& \begin{array}{c}
a=p^{\alpha_{1}} a_{1},\left(a_{1}, p\right)=1, \\
b=p^{\beta_{1}} b_{1},\left(b_{1}, p\right)=1, \\
(c, p)=1,(d, p)=1,(n, p)=1
\end{array}
\end{aligned}
$$

Данная скобка больше $1 / 2$, если $(-c d)$ - квадратичный вычет по модулю $p$; больше 1 и стремится к 1 с ростом $p$, если $(-c d / p)=-1$.

4.4. Случай, когда три коэфбичиента делятся на $p,(n, p)=1$. Пусть

$$
\begin{array}{clll}
a=p^{\alpha_{1}} a_{1}, & \left(a_{1}, p\right)=1, & b=p^{\beta_{1}} b_{1}, & \left(b_{1}, p\right)=1, \\
c=p^{\gamma_{1}} c_{1}, & \left(c_{1}, p\right)=1, & (d, p)=1, & (n, p)=1 .
\end{array}
$$

Найдем $\Phi(p)$ :

$$
\Phi(p)=\frac{1}{p}\left(\frac{d}{p}\right) S(p, 1,0) \sum_{\substack{l=1 \\(l, p)=1}}^{p}\left(\frac{l}{p}\right) e^{-2 \pi i n l / p} .
$$

Используя утверждение 3.1 леммы 3, получаем

$$
\Phi(p)=\frac{1}{p}\left(\frac{-d n}{p}\right) S^{2}(p, 1,0)=\left(\frac{d n}{p}\right) .
$$

Пусть $\alpha_{1} \leqslant \beta_{1} \leqslant \gamma_{1}$. Из утверждения 2.3 леммы 2 и из утверждения 3.1 леммы 3 получим, что $\Phi\left(p^{\alpha}\right)=0$. Имеем следующий множитель:

$$
\begin{aligned}
& \prod_{p}\left(1+\left(\frac{d n}{p}\right)\right) . \\
& \begin{array}{l}
a=p^{\alpha} 1 a_{1},\left(a_{1}, p\right)=1 \\
b=p^{\beta_{1}} b_{1},\left(b_{1}, p\right)=1 \\
c=p^{\gamma_{1}} c_{1},(c, p)=1 \\
(d, p)=1,(n, p)=1
\end{array}
\end{aligned}
$$

Полученная скобка равна нулю в том случае, если $d n-$ квадратичный невычет по модулю $p$; равна двум, если $d n$ - квадратичный вычет по модулю $p$. 
4.5. Случай, когда все коэффициенты делятся на $p,(n, p)=1$. Уравнение (1) не имеет решения. Тем самым получим утверждения теоремы 1.

Доказательство теорем 2 и 3 проводятся аналогично. В данном случае при вычислении точных формул для функции $\Phi\left(p^{\alpha}\right)$ используются утверждения 2.4 леммы 2 и 3.2 леммы 3.

\section{СПИСОК ЛИТЕРАТУРЫ}

1. Малышев А. В. О представлении целых чисел положительными квадратичными формами// Тр. Мат. ин-та им. В. А. Стеклова АН СССР. - 1962. - 65. - С. 3-212.

2. Estermann T. On Kloosterman's sum// Mathematica. - 1961. - 8. - P. 83-86.

3. Estermann T. A new application of the Hardy-Littlewood-Kloosterman method// Proc. London Math. Soc. - 1962. - 12. - P. 425-444.

4. Hua Loo-Keng Introduction to Number Theory. - Berlin-Heidelberg-New York: Springer-Verlag, 1982.

5. Kloosterman H. D. On the representation of number in the form// Acta Math. - 1926. - 49. - P. 407-464.

Куртова Лилиана Николаевна

Белгородский государственный национальный исследовательский университет

E-mail: kurtova@bsu.edu.ru

Мотькина Наталья Николаевна

Белгородский государственный национальный исследовательский университет

E-mail: motkina@bsu.edu.ru 